\title{
Receiver operating characteristics from nonhuman animals: Some implications and directions for research with humans
}

\author{
BRENT ALSOP \\ University of Otago, Dunedin, New Zealand
}

\begin{abstract}
Reviews of signal detection have largely overlooked the research involving nonhuman animal subjects. Some of this research is presented and reanalyzed here. Plots of receiver operating characteristics show that human and nonhuman signal-detection performance is very similar. The studies emphasize the series of discriminations that comprise signal-detection tasks and illustrate the systematic effects of different methods of arranging payoffs or feedback, of the consistency of that feedback, and of the physical disparity between response alternatives. The data provide some support for recent theoretical accounts that favor a criterion location measure of isobias over the likelihood ratio, but they also suggest that more systematic work is required in this area. Overall, this research supports many contemporary views concerning signal detection, and it provides an alternative way of looking at some recurrent issues. It also suggests that extensions of signal detection to analyze data from other research paradigms require some caution, and it offers directions for complementary research with human subjects.
\end{abstract}

Signal-detection theory (SDT) has had considerable influence in contemporary experimental psychology, and it has attracted a number of major theoretical and empirical reviews (e.g., Dúsoir, 1975; Macmillan \& Creelman, 1990; Swets, 1986a, 1986b). Although these articles have highlighted various strengths and weaknesses in our understanding of signal detection and signal recognition, they have not considered in any depth the substantial body of data from studies using nonhuman animal subjects (hereafter referred to simply as animal subjects). The separation of human and animal research in this area seems to stem primarily from differences in the manner that respective experimenters analyze and conceptualize their studies. To this author's knowledge, there is no strong evidence that human and animal performance differs markedly on signal-detection tasks. Furthermore, there are some advantages to using animals as subjects. First, animal subjects are available for an extensive number of experimental conditions, each composed of several thousand trials. Second, the environment, history, and motivation of animal subjects can be more easily controlled, a situation that is well suited for investigating aspects of signal-detection performance, such as response bias. Finally, there are less obvious opportunities for the effects of some factors (e.g., arranged payoffs) to be con-

The author thanks Mike Davison, Dianne McCarthy, and Tony Nevin for their support and many important discussions over the years, and Steve Gallagher and Victoria Johnstone for their help with this article. Reviewers of an earlier version of this article-in particular, A. A. Wright, D. Blough, and R. D. Luce provided many constructive comments. Correspondence should be addressed to B. Alsop, Department of Psychology, University of Otago, Box 56, Dunedin, New Zealand (e-mail: balsop@psy.otago.ac.nz). founded by other factors (e.g., verbal instructions) with animal subjects.

Perhaps more importantly, animal studies have addressed issues relevant to some of the current applications of signal detection. There has been a trend to conceptualize and analyze the data from a variety of experimental and applied situations within a signal-detection framework (e.g., memory research, psychopharmacology, foraging, quality control, weather forecasting, and radiology). Such extensions are not without potential problems. SDT was designed to provide measures of the psychophysical distance between stimuli from experiments carried out in the rarefied atmosphere of the psychophysics laboratory. Once this rigor is relaxed, it becomes less clear what a signal-detection analysis will actually measure. For example, a hypothetical experiment with younger and older children finds that the latter score better on some recognition memory or object recognition task. This might indicate that the older children are better at remembering or identifying these stimuli. On the other hand, this difference might reflect that the younger children had more difficulty maintaining attention on the task, that they found the verbal or mechanical responses more difficult to remember or easier to confuse, or that they found any feedback, or lack of it, more ambiguous. From this perspective, a signaldetection analysis describes performance across an entire detection task of which the characteristics of the sample stimuli form only a part. When the subjects are as naive as pigeons, researchers are forced to consider detection performance from this broader perspective.

The purpose of the present article is to make some of the results and implications of signal-detection research with animal subjects accessible to a wider audience. In particular, it focuses on the evidence that behavior in a signal- 

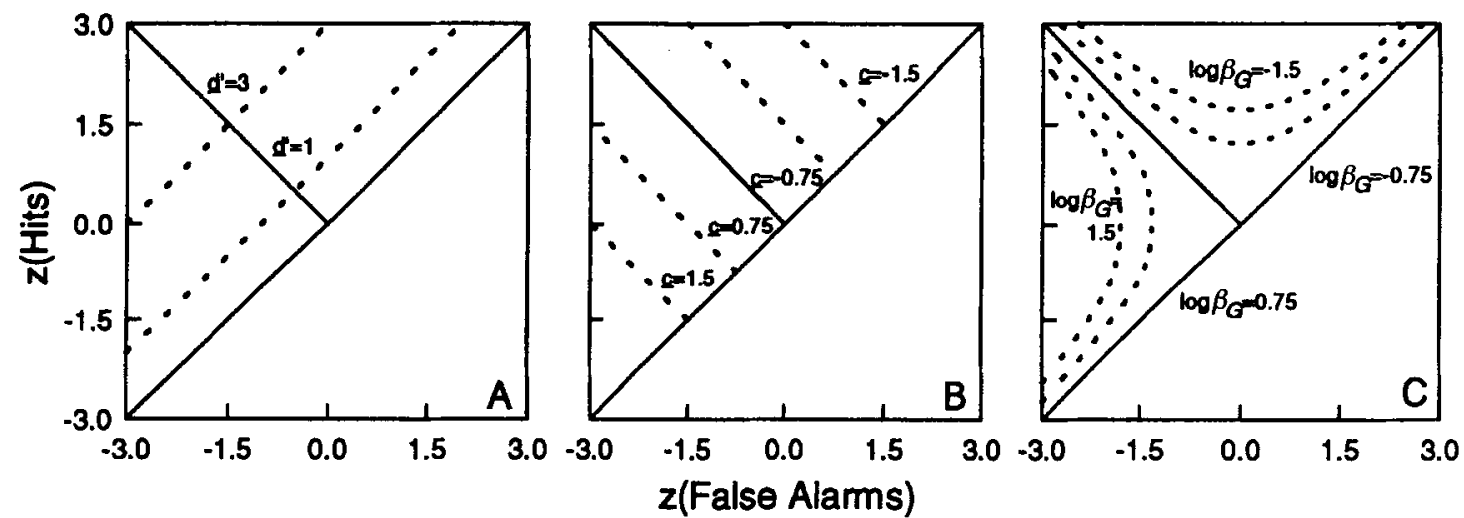

Figure 1. (A) The shape of isosensitivity functions predicted by the $d^{\prime}$ measure of sensitivity in Equation 1. (B) The shape of two equal but opposite isobias functions predicted by using the criterion location measure of bias, $c$. (C) The shape of two equal but opposite isobias functions predicted by the likelihood ratio, $\beta_{G}$.

detection task requires a number of discriminations and that all determine overall performance. It also considers some of the factors that contribute to response bias in detection tasks and the difficulties associated with making meaningful comparisons between estimates of response bias. The article presents the data from a variety of experiments in terms of their receiver operator characteristic (ROC) functions and in relation to the predictions of the prevalent contemporary model of signal detection-that is, the SDT model of Green and Swets (1966).

\section{BACKGROUND}

In a reasonably representative signal-detection procedure, subjects might identify which of two sample stimuli, $S_{1}$ or $S_{2}$, has been presented. The two stimuli might be two values taken from some physical dimension (e.g., different wavelengths of light) or the presence or absence of a stimulus against some background (e.g., "tone + noise" vs. "noise alone"). The subject chooses between two responses, $B_{1}$ or $B_{2}$, to indicate which stimulus was presented. The responses may be mechanical (e.g., hitting one of two keys) or verbal (e.g., "Yes, the stimulus is present," or "No, the stimulus is absent"). Often, the subject receives some feedback or payoff for correct, or sometimes incorrect, responses.

The number of correct responses should provide a simple measure of the discriminability, or sensitivity, between the two stimuli. However, performance is often affected by variables other than the psychophysical distance between $S_{1}$ and $S_{2}$. These variables introduce response bias - that is, a tendency to make more of one type of response than the other, irrespective of the stimulus presented. A response bias can be produced experimentally. For example, if correct $B_{1}$ responses lead to a more valued consequence or "payoff" ( $\$ 50$, say) than correct $B_{2}$ responses $(\$ 1$, say), then performance typically shows a bias for making $B_{1}$ responses. These effects complicate any simple assessment of the subjects' sensitivity on the basis of the number of correct responses alone. Ideally, a model of signal-detection performance should provide independent measures of sensitivity (i.e., the effect of stimulus disparity) and bias (i.e., the effect of other variables). In other words, the measure of sensitivity between a given $S_{1}$ and $S_{2}$ should remain invariant across changes in variables known to produce changes in bias, a condition called isosensitivity (e.g., Swets, 1986b). Similarly, the measure of bias (e.g., produced by unequal payoffs) should remain invariant across changes in variables known to produce changes in stimulus disparity, a condition called isobias (e.g., Dusoir, 1975).

There are various models to describe performance in signal-detection procedures (see, e.g., Macmillan \& Creelman, 1990, 1991; Swets, 1986b). Moreover, some of these models provide more than one measure of bias and, in some cases, sensitivity. The present article will consider only the prevalent measures of bias and sensitivity - that is, those derived from the signal-detection model proposed by Green and Swets (1966). This particular model was selected because earlier reviews and articles indicate that it generally provides better empirical and theoretical accounts of sensitivity and bias than do other models (e.g., Dusoir, 1975; Macmillan \& Creelman, 1990, 1991; Swets, 1986a, 1986b), and, as will be demonstrated later, the empirical form of ROC functions from animal subjects was more consistent with its measures. ${ }^{1}$ Previous authors have described this model in some detail (e.g., Macmillan \& Creelman, 1990; Swets, 1986b); therefore, only a brief account is provided here.

The SDT model of Green and Swets (1966) assumes that each presentation of $S_{1}$ produces a slightly different sensory experience because there are presumabiy momentary fluctuations in the subject's neurophysiology or, in some situations, the surrounding environment. The sensory experience produced by $S_{1}$ is therefore better described by a probability density function. The same is true for $S_{2}$. The extent to which the functions for $S_{1}$ and $S_{2}$ overlap determines the difficulty of the discrimination. 

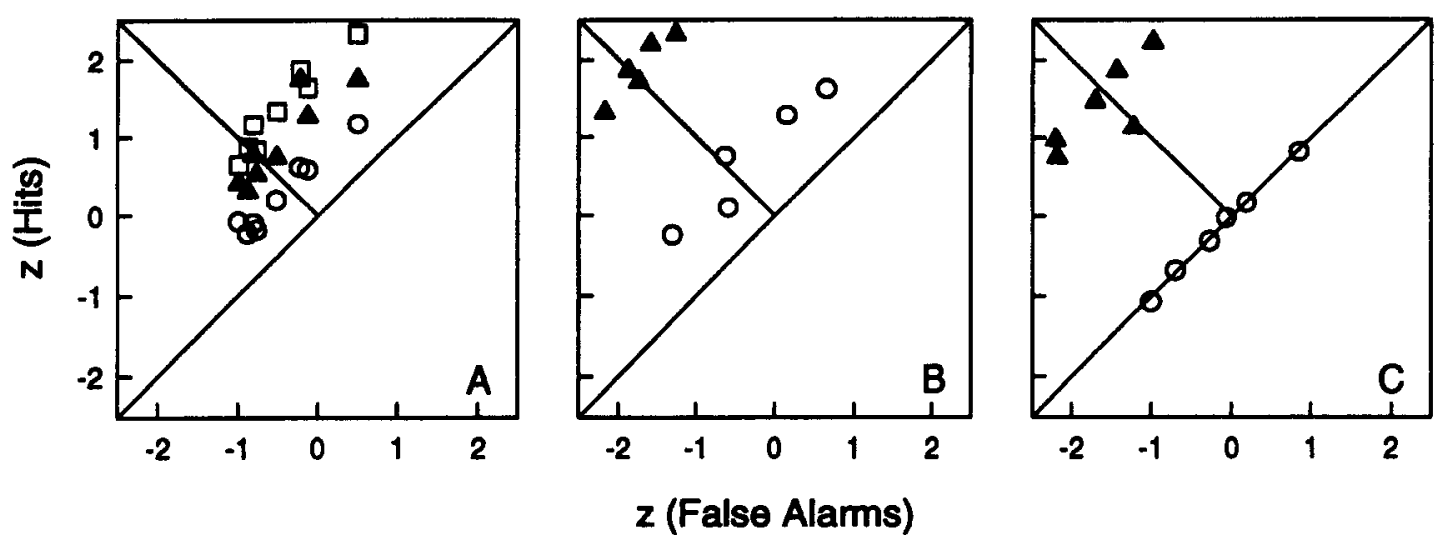

Figure 2. Isosensitivity functions are plotted at levels of sensitivity different from (A) Wright's (1972) same-different task, (B) McCarthy and Davison's (1980) two-alternative choice task, and (C) Davison, McCarthy, and Jensen's (1985) free-operant detection procedure.

While performing the detection task, the subject is a assumed to fix some criterion across these continuous overlapping normal distributions. When a stimulus is presented, the sensory evidence will lie to one or the other side of this criterion and so determines which response is made. In the simplest case, the distributions are normal with equal variance, and sensitivity, $d^{\prime}$, is measured by the difference between the means of the distribution functions. This is found by

$$
d^{\prime}=z(H)-z(F A),
$$

where $z$ is the inverse of the normal distribution function, $H$ is the proportion of $\mathrm{B}_{1}$ responses following $\mathrm{S}_{1}$ presentations (the hit rate), and $F A$ is the proportion of $\mathrm{B}_{1}$ responses following $S_{2}$ presentations (the false-alarm rate). Figure 1A shows the general form of the isosensitivity ROC functions predicted by this measure of sensitivity. When the $z$ transform of the hit rate is plotted as a function of the $z$ transform of the false-alarm rate, the ROC function is a straight line parallel to the major diagonal. The distance of this line from the major diagonal measures the sensitivity, $d^{\prime}$ (Equation 1). This line is called an isosensitivity function because it represents equal measures of sensitivity across different levels of response bias.

Two methods of measuring response bias derived from the SDT model will be considered. The first is the criterion location measure, $c$. This is the distance between the location at which the subject has fixed his or her criterion and the origin (i.e., the crossover point of the two normal distributions). This measure is calculated by

$$
c=-\frac{1}{2}[z(H)+z(F A)],
$$

where all notation is as above. Figure 1B shows the isobias ROC functions predicted by this model under the same conventions as Figure 1A. When the $z$ transform of the hit rate is plotted as a function of the $z$ transform of the false-alarm rate, the isobias ROC function is a straight line parallel to the minor diagonal and at right angles to the major diagonal. The distance of this line from the major diagonal is the measure of bias, $c$ (Equation 2). This line is called an isobias function because it describes the same measure of bias (in this case, $c$ ) across different levels of sensitivity.

A second common measure of response bias associated with SDT is the likelihood ratio, $\beta_{G}$. This measure is the ratio of two normal ordinates, and is given by the equation,

$$
\beta_{G}=\exp \left[-\frac{1}{2} z^{2}(H)\right] / \exp \left[-\frac{1}{2} z^{2}(F A)\right],
$$

where all notation is as above. As noted by Macmillan and Creelman (1990), the relation between $\beta_{G}$ and $c$ is quite simple:

$$
\log \left(\beta_{G}\right)=c d^{\prime} .
$$

In other words, the criterion location multiplied by the sensitivity gives the $\log$ likelihood ratio. Figure 1C shows the isobias curves predicted by the likelihood ratio under the same conventions as Figures 1A and 1B. Unlike the criterion location function, the functions for the likelihood ratio fan out from the minor diagonal as they approach the major diagonal.

\section{EMPIRICAL ROC FUNCTIONS}

\section{Sensitivity}

This section serves two purposes. First, it presents data demonstrating that the form of ROC functions obtained from animal subjects is comparable to those obtained from human observers (e.g., Swets, 1986a) and that these isosensitivity functions can be obtained from a variety of procedures. Second, it demonstrates how some of the other factors present in detection tasks, such as the characteristics of the choice responses and feedback, contribute to the measurement of sensitivity in animal signal-detection tasks. Group data are shown in most figures to conserve space. 
The ROC functions in Figure 2 come from three studies using different procedures to investigate detection performance in pigeons. In each case, the data sets produce orderly functions consistent with the predicted forms of ROC functions shown in Figure 1A. Figure 2A shows the results when a pigeon made same-different judgments concerning different wavelengths of light (Wright, 1972). Two wavelengths of light were presented side by side on the center key of the pigeon's three-key chamber. A subsequent peck on the left key was correct if the two stimuli were identical, and pecking the right key was correct if they differed. Wright varied the probability of reward for the two types of correct response across sessions to produce different response biases. Figure 2A shows representative results from one of Wright's subjects when one of the sample wavelengths was always $640.4 \mathrm{~nm}$ and the other was varied $(635.4 \mathrm{~nm}$, open circles; $633 \mathrm{~nm}$, filled triangles; $629.6 \mathrm{~nm}$, open squares). The orderly isosensitivity ROC plots are similar to those predicted by Figure $1 \mathrm{~A}$ and to those frequently obtained from human observers (e.g., Swets, 1986a).

McCarthy and Davison (1980) trained pigeons in a two-choice discrete-trial procedure. The sample stimuli were two durations of light, and the subjects made their choices by responding on one of two keys following the stimulus presentation: the left key for one duration, and the right key for the other. The payoff distribution for leftkey versus right-key correct responses was varied across conditions to produce response biases. The group data are shown in Figure 2B. The filled triangles trace the isosensitivity contour when the durations were quite disparate $(5 \mathrm{vs} .30 \mathrm{sec})$, and the open circles show performance when the durations were more similar ( $20 \mathrm{vs.} 30 \mathrm{sec}$ ). Again, the data are consistent with the isosensitivity functions shown in Figure $1 \mathrm{~A}$.

Figure $2 \mathrm{C}$ shows the isosensitivity functions obtained from two free-operant, rather than discrete-trial, choice procedures (Davison, McCarthy, \& Jensen, 1985). The sample stimuli were two light intensities. If one of the light intensities was present on the center key, then responses on the left key would eventually lead to a food reward, and responses to the right key would have no consequence. If the other light intensity was present, these contingencies were reversed. The pigeons were free to respond on both keys until the food was obtained. After each food reward, the next light intensity presented was determined at random. The distribution of payoffs was varied across conditions to produce response biases. The number of leftand right-key responses in the presence of each stimulus could be used in a signal-detection analysis. The filled triangles (Figure 2C) show the results of their procedures when the stimuli were distinguishable, and the open circles (Figure 2C) show the corresponding results when the sample stimuli were indistinguishable. Overall, Figure 2 provides good evidence that animal subjects can produce regular ROC plots from a variety of procedures. It is even the case that pigeons' response rates in the presence of related stimuli can be used to construct ROC functions similar to those constructed from the confidence ratings of human subjects (Blough, 1967).

What has become apparent in signal-detection research is that there is not a simple correspondence between the physical and the psychophysical disparity of a pair of stimuli. A number of factors affect ROC plots and corresponding measures of sensitivity and bias, and the effects of some factors are not easily encompassed by detection theory. These factors can be divided into two broad categories: those that involve characteristics of the sample stimuli, and those that involve other aspects of the task. The former category has received considerable attention from researchers involved in both human and nonhuman animal research. For example, estimates of $d^{\prime}$ for a particular pair of stimuli depend on the range of stimulus values presented during the experiment and on the position of that pair within that range (e.g., Braida \& Durlach, 1972), on the amount of discrimination training that the subjects received (e.g., Heinemann \& Avin, 1973), and on the duration of stimulus presentations (e.g., Blough, 1996).

The second category, factors other than characteristics of the sample stimuli, forms a particular focus of this article because the use of animal subjects raises procedural issues that are often taken for granted in human studies. In a variety of experimental situations, animal subjects' asymptotic level of performance is less than would be reasonably expected; in other words, percentages of error responses appear to occur that are independent of the sample stimulus presented. Heinemann, Avin, Sullivan, and Chase (1969) have attributed these error responses, or "guesses," to errors of attention, and this approach has been followed by later researchers. More recently, Blough (1996) has argued that using the term attention is potentially misleading, and he has demonstrated that these guesses can arise as a result of factors not normally associated with attention. He suggested that the source of these guesses was better conceived as a failure in overall stimulus control. This article is in general agreement with Blough's position. The structure of detection and recognition tasks is such that performance can be attenuated in various ways, some of which have little relation to the sample stimuli or to the subjects' attention to those stimuli. However, the term guess is also potentially misleading. Although inattention might lead to an absence of information so that a guess is required (e.g., the subject's eyes might be closed), it is also the case that other parts of the detection procedure require discriminations not unlike those required between the sample stimuli. From this perspective, subjects might fail to perceive factors, such as the difference between the choice responses or the relation between feedback and the choice responses perfectly, or their perceptions might not match the expectations of the experimenter. This could also decrease overall control by the task, and in a manner different from that implied by the term guess. The remainder of this section deals with research that reveals systematic effects illustrating this point. 


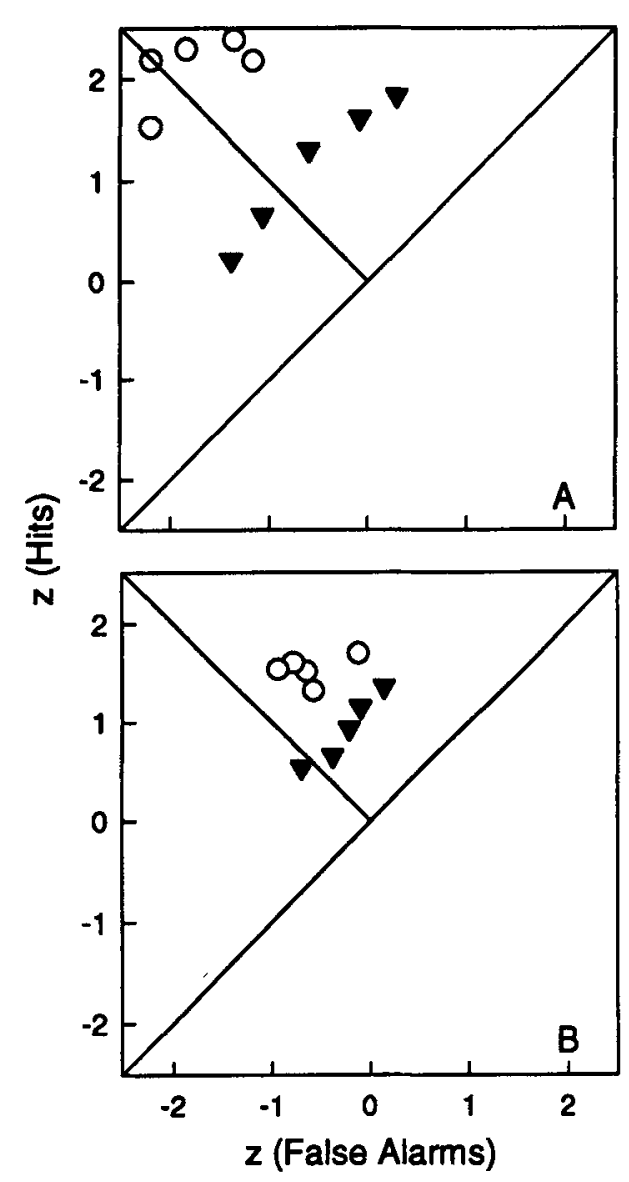

Figure 3. ROC plots showing the effects of varying stimulus disparity and the disparity between the choice responses. In panel $A$, the response disparity was always high, and the stimulus disparity was either high (circles) or low (triangles). Panel B shows the data from the same two levels of stimulus disparity, but with a lower level of response disparity throughout. Data are from Nevin, Cate, and Alsop (1993).

The first procedural factor to be considered concerns the ambiguity or confusability between the responses during the choice phase. Pigeons must be explicitly trained to make the two responses $\left(B_{1}\right.$ and $\left.B_{2}\right)$ in order to identify which stimulus $\left(S_{1}\right.$ and $\left.S_{2}\right)$ was presented. Experimenters usually make the two responses very distinct (different operanda) and assume that there will be minimal confusion between them. Nevin, Cate, and Alsop (1993) explicitly investigated the effect of this response discriminability on signal-detection performance. They arranged two levels of the physical disparity of the sample stimuli (intensities of key light differing by .066 log units and $.032 \log$ units) and two levels of the physical disparity of the choice responses. Choice responses were made to one key, and the two different responses were defined by different response latencies since stimulus offset (at one level, $\mathrm{B}_{1}=0-1 \mathrm{sec}$, and $\mathrm{B}_{2}>2 \mathrm{sec}$; at the other level, $\mathrm{B}_{1}=$ $1-2 \mathrm{sec}$, and $\mathrm{B}_{2}=2-3 \mathrm{sec}$ ). They also arranged a variety of different payoff distributions with each combination of sample stimulus disparities and response disparities by varying the frequency with which their pigeons were reinforced for correct $B_{1}$ and $B_{2}$ responses.

Figure 3 shows the data from Nevin et al.'s (1993) study. The ROCs in Figure 3A show the results when response disparity was higher, and the sample stimuli were more disparate (circles) and less disparate (triangles). Figure $3 \mathrm{~B}$ shows the results for corresponding conditions in which the response disparity was lower. The data for the more disparate sample stimuli tended to be more clustered than the less disparate, but the two important features of this data set are quite clear. First, decreasing the physical disparity between the two response alternatives decreased the measured sensitivity to the two sample stimuli-that is, the ROC plots in Figure 3B are closer to the major diagonal. Second, the data points in Figure 3B are more clustered than those in Figure $3 \mathrm{~A}$ - that is, the physical disparity between the response alternatives attenuated the effects of varying the payoff distribution.

Blough (1996) provides data that are amenable to a similar interpretation. In one two-choice procedure, the pigeons pecked the left key to indicate that a sample stimulus was $568 \mathrm{~nm}$ or less, and they pecked the right key if the sample stimulus was $572 \mathrm{~nm}$ or greater. In another twochoice procedure, the same pigeons identified between the same two sets of stimuli, but they made their choice responses to either a key illuminated by a flickering light or a key illuminated by a steady light, and the position of these two key lights was randomized across trials. Blough plotted the proportion of "greater than $572 \mathrm{~nm}$ " responses as a function of wavelength to produce psychometric functions for both procedures. The asymptotes of the psychometric functions were less extreme for the latter procedure; in other words, the pigeons continued to make a significant number of error responses even when the sample stimuli were very disparate from 568 or $572 \mathrm{~nm}$. Blough interpreted this result as an indication that overall stimulus control was weaker in this part of the study. This could have arisen because there were more errors generated in the translation of the coded stimulus to the choice responses (as Blough suggested) or because the randomized and redundant left-right locations of the flickering and steady key lights increased the confusability or ambiguity between the choice responses.

The second procedural factor concerns the feedback or reward for correct responses. This is another important issue in animal studies of signal detection because the differential consequences for the responses provides the prime source of experimental control. Although it appears that animals will maintain accurate performance at quite low overall rates of reward (McCarthy \& Davison, 1982), the consistency of that feedback is more important. Davison and McCarthy (1980) arranged a signaldetection task where correct identifications of the sample stimuli produced either $3 \mathrm{sec}$ of food with a probability of . 7 or a 3 -sec presentation of the light in the food hop- 


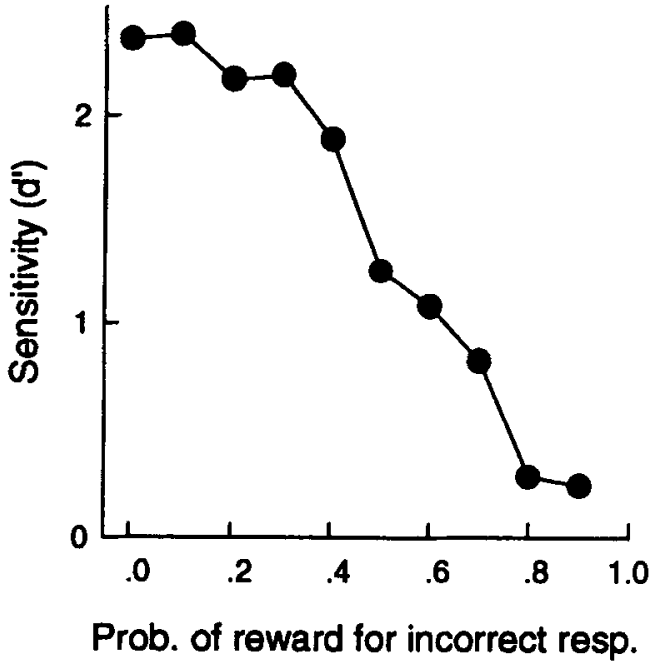

Figure 4. Sensitivity $\left(d^{\prime}\right)$ is plotted as a function of the probability that an incorrect response produced a food reward. Data are from Davison and McCarthy (1980).

per. Incorrect responses produced $3 \mathrm{sec}$ of blackout. Sensitivity was initially high under these conditions (Figure 4). Davison and McCarthy then introduced inconsistent feedback by occasionally rewarding incorrect responses. The probability of a food reward for an incorrect response was progressively varied from .10 to .90 across conditions. As Figure 4 shows, this change produced a decrease in measurements of sensitivity. Furthermore, there were marked changes in sensitivity when the probability of reward for incorrect responses was lower than the probability of reward for correct responses - that is, it was not necessary that the feedback be completely inconsistent to attenuate performance.

Another means of increasing feedback ambiguity is to arrange a delay between the choice response and the subsequent presentation of the reward or feedback. Figure 5 shows illustrative results from a study by McCarthy and Davison (1991). The open circles plot the results from three conditions where the pigeons made their choices immediately after the presentation of the sample stimuli, and correct responses were occasionally followed by an immediate food reward. The distribution of rewards for the two correct responses was varied across the three conditions (ratios of 1:9, 1:1, and 9:1). The open triangles show the results from three similar conditions, but now there was a 25 -sec delay between the offset of the sample stimulus and the presentation of the choice keys ( $\mathrm{S} \rightarrow \mathrm{C}$ delay). Any food rewards were presented immediately following the choice response. This, of course, is a type of recognition memory procedure - a task easily conceptualized and analyzed from a signal-detection framework for both human and nonhuman subjects (e.g., Snodgrass \& Corwin, 1988; Wixted, 1993). The data show the expected drop in measured sensitivity. The filled triangles in Figure 5 show the results when the choice phase immediately followed the sample stimuli, but now any food rewards were delayed by $25 \sec (C \rightarrow R$ delay). The effects of the $\mathrm{C} \rightarrow \mathrm{R}$ delay were very similar to those of the $\mathrm{S} \rightarrow \mathrm{C}$ delay. Delaying the consequences decreased measured sensitivity, and the results were reasonably consistent with the shape of the isosensitivity functions predicted by SDT (Figure 1A). There were, however, some differences between the effects of the two types of delay. The $\mathrm{C} \rightarrow \mathrm{R}$ delay produced a slightly smaller decrease in sensitivity than did an identical $S \rightarrow C$ delay. This difference might have arisen because the $S \rightarrow C$ delay intruded directly on the sample stimulus phase of the task, whereas the effects of the delay that increased the ambiguity of the feedback were more removed. There was also some evidence that the payoff distributions had less effect on performance when the $\mathrm{C} \rightarrow \mathrm{R}$ delay was arranged; these data points were more clustered around the minor diagonal than were the data from the procedure with no delays. This too seems intuitively reasonable. If the relationship between the response alternatives and feedback is less clear, then feedback should have less effect in producing response biases.

It is interesting to compare the results in Figure 5 with those discussed earlier from Nevin et al.'s (1993) study. A delay period between the offset of the stimulus and the opportunity to make a choice response produces effects similar to those produced by decreasing the physical disparity between the sample stimuli-that is, there is a decrease in measured sensitivity. A delay period between the choice response and the consequences for that response produces effects similar to those produced by decreasing the physical disparity between the two responses- that is,

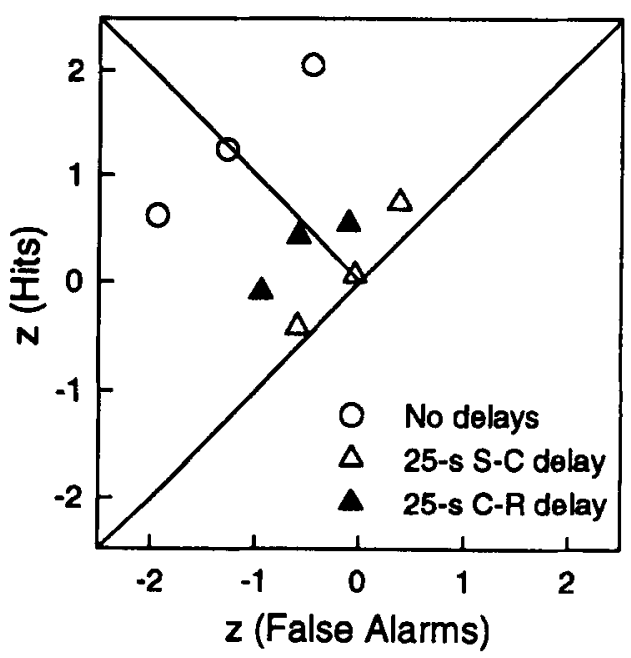

Figure 5. ROC plots of data from delayed-detection procedures. The open circles show the results when there was no delay between stimulus offset and presentation of the choice keys and no delay between completing the choice response and receiving scheduled food reward. The open triangles show the effect of a 25 sec delay between stimulus offset and choice. The filled triangles show the effects of 25 -sec delay between choice responses and scheduled food rewards. All data are from McCarthy and Davison (1991). 


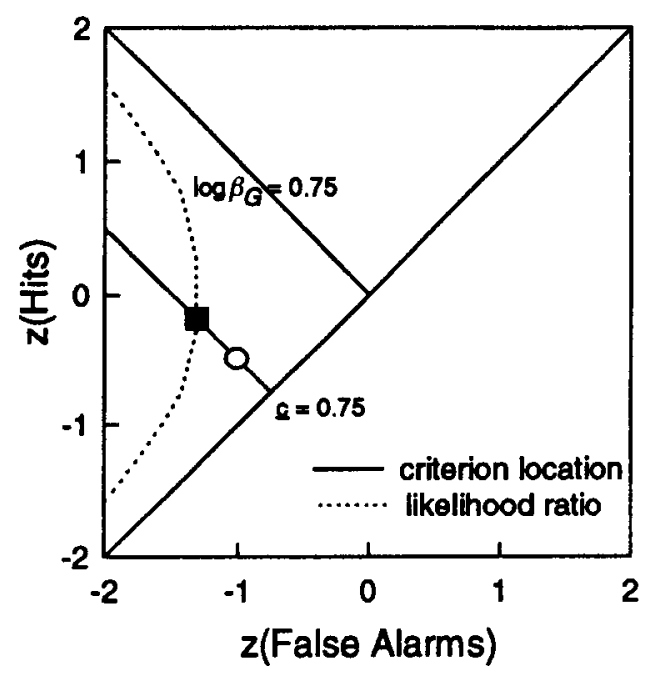

Figure 6. Two hypothetical data points (square and circle) are shown in relation to the predictions of criterion location and likelihood ratio bias measures. See text for details.

there is a decrease in measured sensitivity and a decrease in the effects of payoff manipulations on response bias. These results form a coherent conceptual package, with the effects of delays mimicking the effects of changes in the physical disparity between stimuli or responses, and this strengthens the rationale for a signal-detection approach to recognition memory tasks. The only exception is that McCarthy and Davison (1991) found evidence that increases in the $\mathrm{S} \rightarrow \mathrm{C}$ delay also attenuated response bias, albeit to a lesser extent than corresponding $\mathrm{C} \rightarrow \mathrm{R}$ delays, and there is no parallel result when the physical disparity of sample stimuli is changed directly. This implies that the $S \rightarrow C$ delay also affected control by the choice responses and their consequences. Blough (1996) reports a similar result in a delayed discrimination task. The pigeons were shown a sample stimulus, the stimulus was removed, and after $0,1,2$, or $4 \mathrm{sec}$ had elapsed, they were rewarded for pecking the key showing a rectangle if the sample stimulus was less from the green end of the continuum and for pecking the key showing a disk if the sample was from the blue end of the continuum. He constructed psychometric functions across the wavelength dimension for performance at each delay interval. The asymptotes of the psychometric functions decreased as the delay between sample stimulus and the choice response increased. This implies that overall control was attenuated by the delays. Once Blough corrected for this change in overall control, it was unclear whether the delays had had any effect on sensitivity (i.e., $d^{\prime}$ ) per se.

Taken together, the results of these studies highlight the importance of aspects of the signal-detection procedure other than the sample stimuli themselves. These results emphasize that signal-detection performance is a succession of discriminations between the sample stimuli, between the response alternatives, and between the sources of feedback or reward. Estimates of sensitivity reflect performance across all these levels. A veridical measure of the psychophysical distance between the sample stimuli would require that all other discriminations be perfect. But to what extent is this a problem for signal-detection analyses generally? As suggested earlier, such factors as response disparity and feedback ambiguity are probably sufficiently well defined in a controlled laboratory setting as to be disregarded, but they could seriously confound results in less rigorous settings. Furthermore, it is not necessary that any one of these factors has a dramatic influence on performance by itself; the cumulative effect of small differences in attention, response ambiguity, and feedback ambiguity between two groups could produce quite marked differences in performance. Therefore, an element of caution is required when using signal-detection analyses, and it is not sufficient that experimental data can be analyzed in that fashion.

\section{Bias}

If research requires accurate measures of the psychophysical distance between two stimuli, then response bias is an inconvenient and, ideally, irrelevant phenomenon. However, the question of the sources of response bias and their measurement remains an important issue for signal detection. A complete theoretical account of signal detection requires an adequate understanding of both sensitivity and bias. Furthermore, with the wider application of detection analyses, bias measures are often treated as meaningful dependent variables in their own right. This can be problematic. If two groups show similar sensitivity but different estimates of response bias, then it is probably fair to conclude that the independent variable has produced greater response bias in one group. If, however, the two groups have different sensitivities and different levels of response bias, then it becomes more difficult to interpret the results. First, and as discussed in the previous section, the results might reflect a confound related to the confusability of the choice responses or feedback. Second, the result will depend on the particular model of response bias chosen to analyze the data. Figure 6 illustrates this point. The filled square plots the hypothetical hit rate and false-alarm rate for Group A, and the open circle shows the corresponding result for Group B. If the researchers choose the criterion location measure of bias, $c$ (Equation 2), they would report no difference in response bias between the groups. If they choose the likelihood measure of response bias, $\beta_{G}$ (Equation 3), then they would conclude that there were lower levels of response bias in Group B. There seems to be no established protocol for dealing with this type of situation. A better understanding of the sources of response bias and their relation to models of bias would clarify this problem and could avoid misinterpretations of data.

Macmillan and Creelman (1991) argued that models of bias can be held to three standards. First, the isobias function predicted by a model should be supported empirically. Second, the bias measure should satisfy the monotonicity condition - that is, (1) if 2 subjects have the 

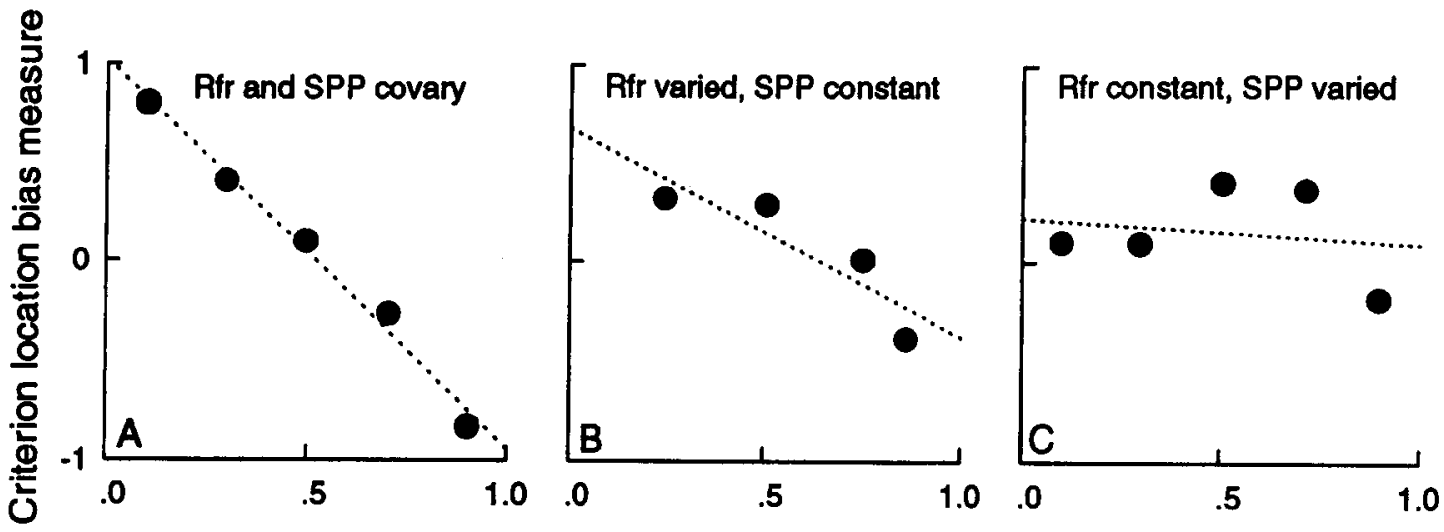

Relative SPP or reinforcer distribution

Figure 7. Measures of response bias are plotted as a function of variations in either the signal presentation probability (SPP; panels $A$ and C) or the reinforcer distribution (Rfr; panel B). See text for details. The data are from McCarthy and Davison (1979).

same $H$ but different $F A$, then the subject with the higher $F A$ shows the greater bias toward making $\mathrm{B}_{1}$ responses, and (2) if 2 subjects have the same $F A$ but different $H$, then the subject with the higher $H$ shows the greater bias toward making $\mathrm{B}_{1}$ responses. This constraint requires that an assessment of bias relies on performance from both $S_{1}$ and $S_{2}$ trials. Third, the measure of response bias should be independent of the measure of sensitivity. Macmillan and Creelman provided a rationale for favoring the criterion location (Equation 2) over the likelihood ratio for the second and third of these standards. However, the first of these standards, empirical evidence, has proved elusive in contemporary research with human subjects (e.g., Dusoir, 1983). In contrast, response bias has been a major research focus in studies of animal performance in signal-detection tasks. The theoretical approach in animal studies has been rooted in the behavioral models of choice, such as the matching relation (e.g., Baum, 1974; Herrnstein, 1970), and the methods of analysis derived from this approach are unlike those normally associated with signal detection. This difference has probably contributed to the distance between human and animal signal-detection research. While some broad concepts involved in the behavioral account of signal detection will appear in this article, a formal account of that model is unnecessary. Interested readers should refer to Davison and Tustin (1978), McCarthy and Davison (1981), or Davison and McCarthy (1988) for a detailed treatment of this approach.

Signal presentation probability and payoff distributions as determinants of response bias. $A$ common method of producing response bias in human signaldetection experiments varies the probability with which the two sample stimuli $\left(\mathrm{S}_{1}\right.$ and $\left.\mathrm{S}_{2}\right)$ are presented. For example, if $\mathrm{S}_{1}$ appears on a greater number of trials (e.g., $80 \%$ ), subjects' responding tends to show a bias toward the $\mathrm{B}_{1}$ response. Conversely, if $S_{2}$ appears on a greater number of trials, subjects' responding tends to show a bias toward the $\mathrm{B}_{2}$ response. Researchers using animal subjects have suggested that stimulus presentation probability, in and of itself, is an ineffective means of producing bias (e.g., Davison \& McCarthy, 1988; McCarthy \& Davison, 1979 , 1981). They contend that this variable is effective only because other aspects of the procedure covary with signal presentation probability. In particular, when $S_{1}$ is presented more frequently than $S_{2}$, then $B_{1}$ responses should typically produce a greater amount of positive feedback or payoff than $\mathrm{B}_{2}$ responses over the course of the session. McCarthy and Davison argued that this confounding variable produces the biased responding, and signal presentation probability alone does not. To test this hypothesis, McCarthy and Davison (1979) trained a group of pigeons in three different procedures. In one procedure, signal presentation probability varied across conditions, and, on average, every third correct response produced a food reward. This meant that the obtained distribution of food payoff covaried with the changes in signal presentation probability. In another procedure, signal presentation probability was held constant but the distribution of food payoff was varied across conditions. In the third procedure, the distribution of food payoff was held constant and signal presentation probability varied across conditions. Figure 7 summarizes the results of this study by plotting the obtained estimates of response bias $(c)$ as a function of the appropriate independent variable. Systematic changes in response bias were obtained in the first two procedures (Figures 7A and 7B), but varying signal presentation probability alone had no orderly effect on performance (Figure 7C). This lack of orderly effect of signal presentation probability precludes some other interpretations of the relationship between response bias and signal presentation probability and payoff distributions in this study. For example, if the response bias were determined by differences in the local rate or conditional probabilities of payoff for $\mathrm{B}_{1}$ and $\mathrm{B}_{2}$ responses, then $\mathrm{Mc}$ - 
Carthy and Davison should still have found systematic effects when the payoff distribution was held constant and the signal presentation probability was varied, because this manipulation varies such factors.

McCarthy and Davison's (1979) result appears at odds with usual ideas about the role of signal presentation probability on detection performance, and there is a temptation to dismiss this effect as a peculiarity associated with a nonhuman species. However, several studies using human subjects have also found somewhat paradoxical results when signal presentation probability was manipulated (Alsop, Rowley, \& Fon, 1995; Johnstone \& Alsop, 1996; Tanner, Haller, \& Atkinson, 1967; Tanner, Rauk, \& Atkinson, 1970). In Tanner et al.'s (1970) study, human subjects identified two tones, and signal presentation probability was varied across conditions. In the first set of conditions, the subjects were experimentally naive, and they were given no instructions concerning the arranged signal presentation probability and no feedback during the experiment. There was a consistent response bias toward the stimulus presented least often. Once the subjects were provided with instructions and feedback, this effect reversed, and there was a consistent response bias for the stimulus presented most often. The instructions and feedback were then removed. The direction of the response biases reversed again and favored the least presented stimulus. Alsop et al. (1995) used human participants in a systematic replication of McCarthy and Davison's (1979) study. They arranged three procedures: (1) Signal presentation probability was varied across conditions, and the payoff distribution was equal and constant, (2) signal presentation probability was equal and constant, and the payoff distribution was varied across conditions, and (3) signal presentation probability was varied across conditions, and the payoff distribution was allowed to covary. The second and third procedures produced changes in response bias consistent with those produced by McCarthy and Davison's animal subjects, but the first procedure produced results different from their study and consistent with those of Tanner and his colleagues (Tanner et a!., 1967; Tanner et al., 1970). When signal presentation probability was varied across conditions and the payoff distribution was held constant and equal, human subjects showed consistent biases for the response associated with the stimulus presented least often. Macmillan and Creelman (1990) suggested that such an inverse effect might arise because subjects tend to believe that the presentation probabilities are equal. Although this is possible, it is difficult to reconcile all the data with such an interpretation. Tanner et al.'s (1970) subjects continued to show an inverse relation between signal presentation probability and response bias, albeit attenuated, when the nofeedback conditions were reinstated and after they were quite experienced with unequal signal presentation probabilities. Furthermore, Johnstone and Alsop (1996) found the size of this effect appears to increase as sensitivity increases. This is at odds with Macmillan and Creelman's interpretation because the subjects should have better evidence that the signal presentation probability is unequal when sensitivity is greater.

The results of the human and animal studies challenge the traditional role of signal presentation probability as a means to produce response bias in detection procedures, but there is also an obvious difference between the human and pigeon data: Humans show an inverse relation between signal presentation probability and response bias, whereas pigeons show no effect. There are two immediate explanations for this difference. First, it might be that the underlying mechanism guiding response bias is different across these species; for example, pigeons might be sensitive to the rates of payoff on each response alternative, whereas humans might be sensitive to the conditional probabilities of payoff. Second, the absence of payoff on certain trials might affect the two species differently; for example, the humans might find the absence of payoff more aversive during such tasks (i.e., no feedback is more like being told you are "wrong") than pigeons do. Such a difference need not arise from separate underlying processes across these species, instead it might reflect differences in their preexperimental histories.

The implication of these experiments for human research is most easily posed as a question: What is the appropriate independent variable for studying the empirical form of isobias functions in human subjects? Perhaps the diversity of bias models in this area (Macmillan \& Creelman, 1990, 1991) reflects a failure to isolate and control the factors that determine bias, rather than an inability to develop a coherent account of this process. One factor traditionally believed to produce response biases (i.e., signal presentation probability) might not do so, or at least it has effects not easily interpreted. This situation becomes more complex because variables such as payoff distributions can be more difficult to control than factors such as signal presentation probability, as the next section will demonstrate.

Arranged and obtained payoff distributions as determinants of response bias. Signal presentation probability is a convenient independent variable because the subject is guaranteed to receive the signal presentation probability that the experimenter arranges (given that the experiment is conducted properly). Obtained payoff distributions, on the other hand, can differ markedly from that which the experimenter arranges. Consider a procedure in which the difference between the two stimuli is quite small (i.e., the number of errors is quite high), signal presentation probability is .80 , say, and all correct responses are rewarded in some fashion (i.e., feedback/ points). Under these conditions, the payoff distribution is unlikely to be the same as the signal presentation probability. As the subject begins to show a response bias for the response alternative with a greater payoff rate (i.e., that which is correct for the more frequently presented stimulus), the number of rewarded responses on that alternative should increase, whereas the number of rewarded 

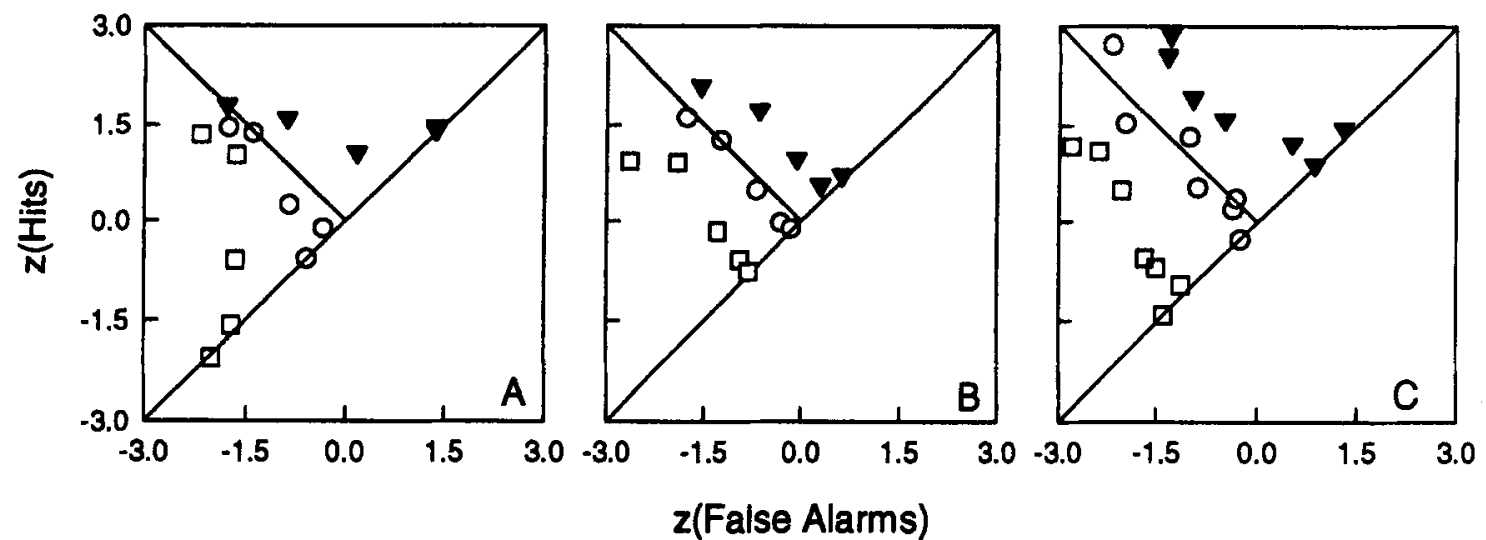

Figure 8. Isobias data from (A) the uncontrolled payoff distribution procedure of McCarthy and Davison's (1984) study, (B) the controlled payoff distribution procedure of McCarthy and Davison's (1984) study, and (C) the controlled payoff distribution procedure of Alsop and Davison's (1991) study. The triangles and squares show results from conditions with unequal payoff distributions; the circles show the results from conditions with equal frequencies of payoff across alternatives.

responses on the other alternative will decrease. This creates a more extreme payoff distribution than the signal presentation probability arranges. In principle, this new obtained payoff distribution can feedback onto the subject's performance to produce a larger response bias and an even more extreme payoff distribution. Presumably, this cycle continues until it is constrained by other factors, such as marked decreases in the overall payoff rate. This means that the experimenter no longer has control over a potent experimental variable. The extent to which this is a problem for signal-detection performance is also likely to be a function of the disparity between the stimuli. When the difference between the stimuli is greater, the subjects make fewer errors in total, and so the obtained and arranged payoff distributions should be more similar. This effect will contaminate any empirical isobias data generated by this procedure.

Other procedures share this problem. For example, an experimenter might arrange equal numbers of stimulus presentations (i.e., signal presentation probability $=.50$ ) but arrange unequal payoffs by rewarding every second correct response on average following $S_{1}$ presentations but only every fourth correct response on average for the other class of correct responses. Again, the subject's performance will determine the obtained payoff distribution in such a procedure. The same effect can occur if the arranged signal presentation probability and reinforcer distributions are equal, but the size of the payoffs is different for the response alternatives. In all these situations, as subjects develop a response bias for an alternative, then they will receive more payoffs for that alternative and less on the other. McCarthy and Davison $(1981,1984)$ used the term uncontrolled reinforcer ratio procedure to describe experiments that allow payoff distributions to covary with performance in this manner.

McCarthy and Davison (1984) demonstrated that different ROC functions were obtained when the experimenter arranged uncontrolled and, as will be described below, controlled payoff distributions. In their uncontrolled procedure, 6 pigeons were trained to discriminate between two intensities of light $\left(S_{1}\right.$ and $\left.S_{2}\right)$ by choosing between two response keys $\left(B_{1}\right.$ and $\left.B_{2}\right)$. The signal presentation probability was varied across three conditions $(.20, .50$, and .80$)$, and every third correct response on average was rewarded. McCarthy and Davison then changed the stimulus disparity between the two intensities of light and repeated the signal presentation probability manipulations. In all, this procedure was conducted for five levels of stimulus disparity. The results were quite orderly, and they were interesting on two counts. First, the obtained payoff distribution deviated from the arranged distribution as a function of stimulus disparity for the two asymmetric signal presentation probability conditions $(.20$ and .80 ). This deviation was consistent with the prediction above: The obtained payoff distributions were more extreme when stimulus disparity was smaller than that obtained when the stimulus disparity was larger. Second, the ROC plots (Figure 8A) show that the data from the two unequal signal presentation probability conditions followed a pattern somewhat similar to that described by the likelihood ratio measure of bias (Equation 3, Figure 1C), fanning out from the minor diagonal as sensitivity decreased. This pattern was also evident in the results of the individual subjects.

In their controlled payoff distribution procedure, McCarthy and Davison (1984) used the same 6 pigeons and the same five levels of stimulus disparity. The signal presentation probability was always set at .50, however, and the payoff distribution was varied across three conditions $(.20, .50$, and .80$)$ at each level of stimulus disparity. The payoffs were arranged in a probabilistic fashion such that the subject was constrained to receive the same distribution of payoffs as that arranged by the experimenter. The group data are shown in Figure 8B. The results were quite orderly, but the data from the conditions with unequal payoff distributions now produced response biases more 
consistent with the predictions of the criterion location measure of bias (Figure 1B) than those of the likelihood ratio. These results are supported by another large systematic study. Alsop and Davison (1991) arranged seven levels of stimulus disparity, and, at each level, they varied the controlled payoff distribution that the pigeons received across three conditions $(.12, .50$, and .88). Their group results (Figure 8C) were similar to McCarthy and Davison's (Figure 8B), albeit more variable, and they were more consistent with the pattern predicted by the criterion location measure of bias because they failed to show the extreme deviations from the minor diagonal that are predicted by the likelihood ratio (Figure 1).

Corresponding data from human subjects are limited. Dusoir (1983) conducted what is probably the most extensive study of this type with humans. However, his experiments used variants of uncontrolled proceduresthat is, although the experiment arranged asymmetric sizes or frequencies of the payoffs, the final payoff distributions were determined by performance. This might explain the considerable differences between individual subjects' ROC plots, from which Dusoir concluded that his subjects' response bias was determined by a number of individual criteria. Recent work by Johnstone (1998), who conducted a systematic replication of McCarthy and Davison's (1984) procedure using human subjects, has been more promising. The subjects received conditions with both controlled and uncontrolled payoff distributions and four levels of stimulus disparity. The mean ROC plot produced results very similar to those shown in Figure 8 , in terms of both the absolute level of the response biases and the different patterns generated by the controlled and uncontrolled procedures. As expected, there was a consistent relation between the obtained payoff distributions that the subjects received and the ROC data throughout the study. Overall, it appears that controlled and uncontrolled payoff distributions can account for some of the major differences in patterns of response bias found in both human and animal studies.

This research supports the idea that a major barrier toward our understanding of response bias has been isolating and controlling the various sources of bias in signaldetection procedures (Macmillan \& Creelman, 1991). An experiment can generate data consistent with either of two competing signal-detection models of response bias simply by changing the manner in which the payoffs are arranged. A further implication of these studies is that if the sources of bias are adequately controlled, then orderly isobias functions are obtained, and these isobias functions approximate the pattern predicted by the criterion location model of response bias.

\section{IMPLICATIONS AND DIRECTIONS}

The present article largely ignores the conceptual differences in the manner that researchers using human subjects and those using animal subjects have approached signal detection, and this empirical approach highlights areas where studies with animals can play a useful role in our understanding of signal-detection performance. This research has important implications for the general use of detection theory, and it demonstrates that extensions of signal-detection analyses to other research areas require caution. Ultimately, signal-detection performance is the product of a variety of discriminations involving the sample stimuli, the response alternatives, and the feedback or outcomes for those choices. In this sense, all detection tasks require serial information processing, and what is measured as sensitivity and response bias depend on the accuracy of all the discriminations. Of course, these factors must be coupled with more common concerns, such as the influence of the general attention of the subjects, the presentation time of the stimuli, and the effects of any delays during the task (e.g., Blough, 1996; McCarthy \& Davison, 1991).

It is difficult to determine post hoc whether a signaldetection analysis is measuring exactly what the experimenter expects. This is especially true for experiments that compare the performance between two groups at one level of an independent variable (say, the recognition memory of two age groups of children) or one group at two levels of an independent variable (say, detection performance by pilots with and without induced hypoxia). In some cases, it might be possible to use a range of stimuli as $S_{1}$ (a number of softer tones, say) and $S_{2}$ (a number of louder tones, say), then there would be sufficient data to construct a psychometric function for each group. For many stimulus dimensions, the asymptotic performance of two groups should be similar, even if one group reaches those asymptotes more quickly than the other. If asymptotic performance of one group is less than the other group, then this suggests that overall control, rather than (or as well as) control by the sample stimuli, has been attenuated (e.g., Blough, 1996). Heinemann, Chase, and their colleagues have developed an analysis called correction for guessing, or correction for inattention, to make comparisons between such psychometric functions more meaningful (e.g., Heinemann et al., 1969). This analysis recalibrates the response proportions obtained for each stimulus so that the data from both groups extend over the entire range of the $y$-axis, and then comparisons of the slopes and mid points of the psychometric functions should be more meaningful. Unfortunately, this style of experiment will not be amenable to all experimental and applied situations.

Manipulations designed to produce response bias can also reveal whether response or feedback factors are attenuating performance. The effects of payoff manipulations should be less extreme than expected if response or feedback are not clearly defined. In many contexts, however, it would be difficult to decide what constitutes "less extreme" in the absence of an appropriate comparison. Cer- 

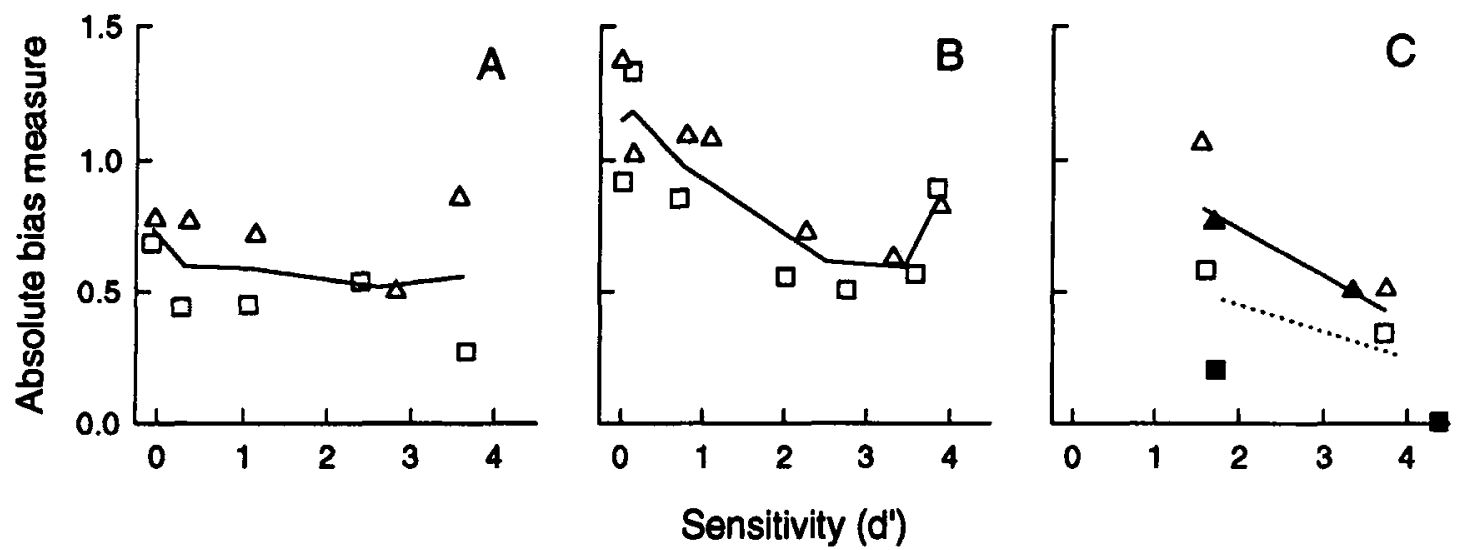

Figure 9. Estimates of bias $(c)$ are plotted as a function of their corresponding estimates of sensitivity for the data shown in Figures $8 \mathrm{~B}$ and $8 \mathrm{C}$ (panels $\mathrm{A}$ and $\mathrm{B}$ ) and Figure 3 (panel $C$ ). The triangles and squares in each panel represent conditions with unequal payoff distributions (e.g., 1:9) and their reversals $(9: 1){ }^{2}$ The lines show the averages of these pairs of corresponding conditions. In panel $C$, the open and filled symbols show the results when response disparity was high and low, respectively.

tainly, if one group of subjects shows lower sensitivity and less effect of payoff manipulations, the researchers should consider that factors unrelated to the sample stimuli might contribute to the lower sensitivity.

Although this is a problem for measuring sensitivity, even more caution is necessary if measures of response bias are to be compared in a meaningful way. First, there is no consistent protocol for choosing one measure of response bias over another. Researchers can, intentionally or not, produce or remove differences in response bias between subjects according to the measure that they adopt (e.g., Figure 6). This problem is compounded since the results of some uncontrolled procedures will be unamenable to reasonable analysis by any measure of bias because there has been limited control of independent variables, such as the obtained payoff distributions between subjects (i.e., uncontrolled payoff procedures) in these cases.

In human detection experiments, verbal instructions can be used to produce response bias - that is, the subjects can be instructed to adopt a lax or strict criterion. There is no direct equivalent in animal procedures, so this type of procedure is largely outside the scope of the present article. That said, although this technique can be useful for generating isosensitivity functions from a human subject, it does not overcome many of the problems outlined above: The detection task remains a series of potentially imperfect discriminations. Furthermore, verbal instructions are a difficult base from which to build quantitative accounts of detection performance.

On the other hand, these animal studies also offer positive contributions for the wider use of a signal-detection approach. A broader appreciation of the factors that influence detection performance can increase its usefulness as an applied tool, especially in terms of applied problems not normally framed as signal-detection tasks. For example, a program to prevent child abduction can be conceptualized as a form of signal-detection training. Suc- cessful programs must focus on teaching children not only to distinguish between risk and no-risk situations but also to select between clear response alternatives in these situations (e.g., Poche, Yoder, \& Miltenberger, 1988). Furthermore, it is important to maintain consistent feedback, even if a "false alarm" in such an applied situation could lead to some embarrassment or inconvenience. Similarly, these considerations offer directions for improving performance in applied tasks requiring testing of samples or quality control (e.g., Mason \& Redmon, 1992).

This research with animals also makes a contribution to understanding signal detection and signal recognition at a theoretical level. Swet's (1986a) review of the empirical form of isosensitivity functions favored SDT over other competing models, and the data from animal subjects are generally consistent with that finding. Similarly, Macmillan and Creelman's $(1990,1991)$ reviews favored criterion location measures of response bias over likelihood ratio models on pragmatic and theoretical grounds, and the research with animals provides empirical support for that theoretical position. Furthermore, animal studies suggest that the variety of bias models might have arisen from different ways of arranging factors, such as payoffs - that is, different patterns of response bias seem to reflect different procedures rather than different processes. This focus on procedure currently seems the closest approximation to the theory of instructions thought necessary to predict isobias performance (Macmillan \& Creelman, 1991).

Although the data from animals approximate the criterion location measure of bias, there is also a suggestion that there are small, but systematic, deviations from this model as well. Figures $9 \mathrm{~A}$ and $9 \mathrm{~B}$ show a reanalysis of the data from McCarthy and Davison's (1984) and Alsop and Davison's (1991) "controlled" payoff distribution procedures (Figures $8 \mathrm{~B}$ and $8 \mathrm{C}$ ). Estimates of sensitivity $\left(d^{\prime}\right)$ and bias $(c)$ for those conditions that arranged asym- 
metric payoff distributions were calculated and then plotted as a function of each other (the symmetric payoff distributions are uninformative for this analysis). ${ }^{2}$ If the criterion location describes an isobias function for these data, then there should be no systematic changes in response bias as sensitivity increases. Estimates of response bias tend to decrease as sensitivity increases. This pattern was also evident in plots of their individual subjects' data. The data from Nevin et al.'s (1993) study (Figure 3A), which also used a controlled payoff distribution procedure, were analyzed in the same manner. The results from the high-response-disparity conditions are shown in Figure 9C. Although there were only two levels of stimulus disparity in this experiment, the pattern was consistent with the results of McCarthy and Davison (1984) and Alsop and Davison (1991). Overall, the criterion location measure of bias does not provide an isobias measure for these sets of data. A similar conclusion was reached when these data were analyzed using the sensitivity and criterion location measures derived from Luce's (1963) choice theory.

The question for future research is whether ideas concerning response bias need remodeling or reconceptualizing, or whether the role of still other factors in the detection procedure warrants attention. A recent study by Alsop and Rowley (1996) took the latter approach with some success. Pigeons were trained extensively in a signaldetection procedure that varied the payoff distribution across conditions to produce response biases. They recorded the reaction times for each choice response, and they then calculated estimates of response bias for faster and slower reaction times. Response biases were markedly greater for faster trials than for slower trials, a result that is consistent with the limited human research in this area (Lappin, 1978). Furthermore, Alsop and Rowley found that response biases were greater on trials immediately following reward and that these two effects appeared to be additive. If stimulus disparity (and its resultant effects on sensitivity) affects such local variations in signal-detection performance, then this would provide one possible indirect mechanism to account for covariations in sensitivity and response bias.

More generally, the data presented in this article might not necessitate a radical reconstruction of signal-detection theories; rather, the data serve more to support and to elaborate existing ideas. Those challenges to traditional views of detection (e.g., the role of signal presentation probability as a source of response bias, or the relationship between reaction time and response bias) can be readily tested. Indeed, perhaps the most important facet of this article is the directions it provides for complementary research with humans, particularly in those areas where corresponding human data are limited.

\section{REFERENCES}

Atsop, B., \& Davison, M. [C.] (1991). Effects of varying stimulus disparity and the reinforcer ratio in concurrent-schedule and signal-detection procedures. Journal of the Experimental Analysis of Behavior, 56, 67-80.
AlsoP, B., \& ROWLEY, R. (1996). Types of responding in a signaldetection task. Journal of the Experimental Analysis of Behavior, 65 , 561-574.

AlsoP, B., Rowley, R., \& Fon, C. (1995). Human symbolic matching to sample performance: Effects of reinforcer and sample-stimulus probabilities. Journal of the Experimental Analysis of Behavior, 63, 53-70.

BAUM, W. M. (1974). On two types of deviation from the matching law: Bias and undermatching. Journal of the Experimental Analysis of Behavior, 22, 231-242.

BLoUgH, D. (1967). Stimulus generalization as signal detection in pigeons. Science, $158,940-941$.

BLOUGH, D. (1996). Error factors in pigeon discrimination and delayed matching. Journal of Experimental Psychology: Animal Behavior Processes, 22, 118-131.

Braida, L. D., \& Durlach, N. I. (1972). Intensity perception: II. Resolution in one interval paradigms. Journal of the Acoustical Society of America, 51, 483-502.

Davison, M. [C.], \& MCCarThy, D. [C.] (1980). Reinforcement for errors in a signal-detection procedure. Journal of the Experimenial Analysis of Behavior, 34, 35-47.

Davison, M. [C.], \& MCCARTHY, D. [C.] (1988). The matching law: A research review. Hillsdale, NJ: Erlbaum.

Davison, M. [C.], MCCarthy, D. [C.], \& Jensen, C. (1985). Component probability and component reinforcers as biasers of free-operant detection. Journal of the Experimental Analysis of Behavior, 44, 103-120.

Davison, M. C., \& Tustin, R. D. (1978). The relation between the generalized matching law and signal-detection theory. Journal of the Experimental Analysis of Behavior, 29, 331-336.

Dusolr, A. E. (1975). Treatments of bias in detection and recognition models: A review. Perception \& Psychophysics, 17, 167-178.

DusorR, T. (A. E.) (1983). Isobias curves in some detection tasks. Perception \& Psychophysics, 33, 403-412.

GREEN, D., \& SWETS, J. A. (1966). Signal detection theory and psychophysics. New York: Wiley.

HeinemanN, E. G., \& Avin, E. (1973). On the development of stimulus control. Journal of the Experimental Analysis of Behavior, 20, 183-195.

Heinemann, E. G., Avin, E., Sullivan, M. A., \& Chase, S. (1969). Analysis of stimulus generalization with a psychophysical method. Journal of Experimental Psychology, 80, 215-224.

HERRNSTEIN, R. J. (1970). On the law of effect. Journal of the Experimental Analysis of Behavior, 13, 243-266.

JOHNSTONE, V. (1998). A behavioral approach to human signal detection. Unpublished doctoral dissertation, University of Otago.

JOHNSTONE, V., \& ALSOP, B. (1996). Human signal-detection performance: Effects of signal presentation probabilities and reinforcer distributions. Journal of the Experimental Analysis of Behavior, 66, 243-263.

LAPPIN, J. S. (1978). The relativity of choice behavior and the effect of prior knowledge on the speed and accuracy of recognition. In N. J. Castellan, Jr., \& F. Restle (Eds.), Cognitive theory (Vol. 3, pp. 139168). Hillsdale, NJ: Erlbaum.

LUCE, R. D. (1963). A threshold theory for simple detection experiments. Psychological Review, 70, 61-79.

Macmillan, N. A., \& Creelman, C. D. (1990). Response bias: Characteristics of detection theory, threshold theory, and "non-parametric" indexes. Psychological Bulletin, 107, 401-413.

Macmillan, N. A., \& Creelman, C. D. (1991). Detection theory: A user's guide. Cambridge: Cambridge University Press.

MASON, M. A., \& REDMON, W. K. (1992). Effects of immediate versus delayed feedback on error detection accuracy in a quality control simulation. Journal of Organizational Behavior Management, 13, 49-83.

MCCARTHY, D. [C.], \& DAVISON, M. [C.] (1979). Signal probability, reinforcement and signal detection. Journal of the Experimental Analysis of Behavior, 32, 373-386.

MCCARTHY, D. [C.], \& DAvison, M. [C.] (1980). Independence of sensitivity to relative reinforcement rate and discriminability in signal detection. Journal of the Experimental Analysis of Behavior, 34, 273-284.

MCCARThy, D. [C.], \& Davison, M. [C.] (1981). Towards a behavioral theory of bias in signal detection. Perception \& Psychophysics, 29 , 371-382. 
MCCARThy, D. [C.] \& Davison, M. [C.] (1982). Independence of stimulus discriminability from absolute rate of reinforcement in a signaldetection procedure. Journal of the Experimental Analysis of Behavior, 37, 371-382.

MCCARThy, D. [C.], \& Davison, M. [C.] (1984). Isobias and alloiobias functions in animal psychophysics. Journal of Experimental Psy. chology: Animal Behavior Processes, 10, 390-409.

MCCarThy, D. C., \& Davison, M. [C.] (1991). The interaction between stimulus and reinforcer control on remembering. Journal of the Experimental Analysis of Behavior, 56, 51-66.

Nevin, J. A., Cate, H., \& Alsop, B. (1993). Effects of differences between stimuli, responses, and reinforcer rates on conditional discrimination performance. Journal of the Experimental Analysis of Behavior, 59, 147-161.

Poche, C., Yoder, P., \& Miltenberger, R. (1988). Teaching selfprotection to children using television techniques. Journal of Applied Behavior Analysis, 21, 253-261.

Snodgrass, J. G., \& Corwin, J. (1988). Pragmatics of measuring recognition memory: Applications to dementia and amnesia. Journal of Experimental Psychology: General, 117, 34-50.

SwETs, J. A. (1986a). Form of empirical ROCs in discrimination and diagnostic tasks. Psychological Bulletin, 99, 181-198.

SwETs, J. A. (1986b). Indices of discrimination or diagnostic accuracy: Their ROCs and implied models. Psychological Bulletin, 99, 100-117.
Tanner, T. A., JR., Haller, R. W., \& Atkinson, R. C. (1967). Signal recognition as influenced by presentation schedules. Perception $\&$ Psychophysics, 2, 349-358.

Tanner, T. A., Jr., Rauk, J. A., \& Atkinson, R. C. (1970). Signal recognition as influenced by information feedback. Journal of Mathematical Psychology, 7, 259-274.

WIXTED, J. T. (1993). A signal detection analysis of memory for nonoccurrence in pigeons. Journal of Experimental Psychology: Animal Behavior Processes, 19, 400-411.

Wright, A. A. (1972). Psychometric and psychophysical hue discrimination functions for the pigeon. Vision Research, 12, 1447-1464.

\section{NOTES}

1. The signal-detection model derived from Luce's (1963) choice theory makes predictions that are virtually indistinguishable from SDT. This paper cannot separate these models, so only SDT will be considered for the sake of simplicity.

2. The estimates of response bias derived from payoff distributions that predict a negative value for $c$ were multiplied by -1 . This enabled ready comparison of absolute changes in bias as a function of sensitivity.

(Manuscript received May 9, 1996; revision accepted for publication January 5, 1998.) 